\title{
Analyzing the Effect of Temperature on Squash Ball Impacts Using High-Speed Camera Recordings
}

\author{
Bence Ferenc Berencsi ${ }^{1}$, Attila Kossa ${ }^{1 *}$ \\ 1 Department of Applied Mechanics, Faculty of Mechanical Engineering, Budapest University of Technology and Economics, \\ H-1111 Budapest, Múegyetem rkp. 5., Hungary \\ * Corresponding author, e-mail: kossa@mm.bme.hu
}

Received: 18 April 2021, Accepted: 08 June 2021, Published online: 22 September 2021

\begin{abstract}
Description of the impact characteristics of different types of balls has a great importance in sport science and in engineering. The primary objective of the present paper is to investigate the effect of the temperature on the impacts of different types of squash balls from a given company. The shots were performed using a self-built air-cannon. The impacts were recorded by a high-speed camera and the recorded videos were analyzed by an image-processing method based on a background subtraction technique. Summarizing the main dynamical parameters, we can conclude that increasing the initial speed will decrease the contact time, the coefficient of restitution (COR) and the rebound resilience, whereas these parameters increase at elevated temperatures. The compression tests revealed that within the low velocity range the deformation of the ball's material and not the compression of the inner gas is the main contribution in the force needed to compress the ball. However, when the ball suffers large deformations, the internal air pressure has a huge effect on the rebound behavior. The measurements revealed that there is an optimal initial velocity distinct from the maximum one where the rebound velocity of the ball is higher than in all other cases. From the results we can state that the ball's overall stiffness grows as the temperature increases.
\end{abstract}

Keywords

squash balls, squash ball impact, effect of temperature, high-speed camera recordings, compression tests

\section{Introduction}

We can divide the ball sports into three different groups based on their ball types. First, we can distinguish solid ones (golf, cricket), where the ball's behavior mostly depends on its material parameters. There are papers dealing with the topic of solid ball impacts [1-6] and ball impacts in general [7-11]. However, there are balls, where we can conclude that the dynamic behavior is mostly affected by the condition and the amount of the inner gas enclosed in the ball. The material properties have less impact on such balls (e.g. soccer [12]). These balls can be categorized into a second group. Between the presented two groups, there is a transitional category called hollow balls where we can fit in the squash balls. This group typically contains balls with a relatively thick wall that is filled with air or other gas mixes. In the topic of ball collisions, we can find experiments of hollow ball impacts investigating ping-pong and tennis balls [13-21] as well as squash balls [22-23] and there are some which also dealt with the effect of temperature on ball impacts [24-25]. They mainly examined one ball type with limited impact parameters, e.g. regarding the energy loss or the ball's hang time.

In the present article, we investigate all squash ball types from a given company and we analyze the influence of the temperature change on their impact characteristics. Our goal is to fill the gaps of the topic by showing pieces of evidence for some interesting phenomena presented before [22] and to form an overall summary of the dynamics of squash at the same time.

\section{Experimental procedures}

\subsection{Impact tests}

The results presented in this paper were obtained with five different types of squash balls which were purchased at the same time from the same place. Therefore, we can consider that they were in the same condition. For the measurements, we chose the brand called Artengo from Decathlon because they have the same external diameter regardless of the ball type, unlikely to Dunlop. This parameter was 
Table 1 Parameters of the measured squash balls

\begin{tabular}{lccccc}
\hline & & Squash balls' parameters & & \\
\hline Type & Super slow SB960 & Super slow SB930 & Slow SB590 & Medium SB560 & Fast SB190 \\
Color & Black & Black & Black & Black & Blue \\
Dot & 2 yellow & 1 yellow & 1 Grey & 1 Red & Improver \\
Level & Competition & Club & Improver & Beginner \\
Nominal diameter $[\mathrm{mm}]$ & & & 40 & & \\
Nominal mass $[\mathrm{g}]$ & & & 24 & & \\
\hline
\end{tabular}

vital to perform the shots identically using the same setup. We can distinguish five different ball types in this brand from the fastest one to the slowest one. Each ball has a color code with a base color and dots, which we will use to identify them henceforth. The main parameters of the balls are listed in Table 1.

A custom-made air cannon was built for the measurement (see Fig. 1). The air-launcher was calibrated before the actual measurement to match the air pressure values in the tank with the ball velocities which can occur during a real squash match. From this premeasurement procedure, we obtained the values of the air-pressure that was applied identically for every ball shot to reach almost the same ingoing speed. To record the impacts a Photron FASTCAM SA5 High-speed camera was used, equipped with a Nikon Nikkor AF 200mm f/4D IF ED camera lens. We used 20000 FPS (frame per second) for the recordings. The evaluation process was made in Matlab based on a background subtraction method, which allowed us to track the ball through the determination of its centroid.

In case of a real squash game, at the beginning of the match the players start to warm up the ball for a 4 minutes interval as the World Squash Rules documentation mentions [26]. The heated ball's temperature is usually around $45^{\circ} \mathrm{C}$ [23], so we chose $40 \mathrm{C}^{\circ}$ and $50{ }^{\circ} \mathrm{C}$ as nominal values besides the room temperature, which was $30{ }^{\circ} \mathrm{C}$ during

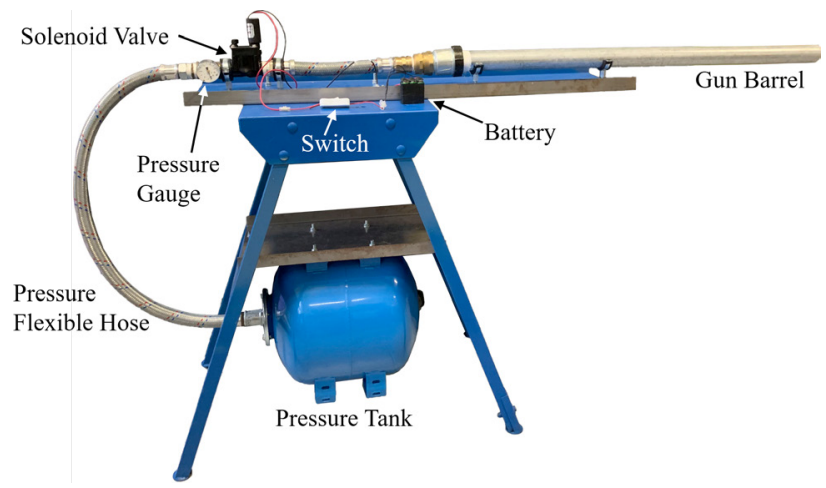

Fig. 1 Air cannon used for the experiments. that time. We performed the shots with one ball type at a certain temperature level with 5 different initial speeds, which means 75 different impacts in total. The temperature of the ball's surface was always checked before and after the impact with a Seek Thermal Compact PRO thermal camera. An example can be seen in Fig. 2.

In general, we can say that there was a $3{ }^{\circ} \mathrm{C}$ temperature drop between the two values (before and after the impact), but it took about 15 seconds to perform a shot and do the measurements.

During the measurements, the air-launcher's tube was horizontal but not completely perpendicular to the surface to avoid the impact of the rebounding ball and the air-cannon but still keep it in the camera's depth of field. The angle was around $88^{\circ}$ between them in the horizontal plane, so it does not influence the quality of the records. The impacts were performed against a steel surface, which was much heavier $(55 \mathrm{~kg})$ and stiffer than the squash ball. In addition, the steel surface was fixed to the wall.

\subsection{Compression tests}

On the other hand, we also carried out compression tests using an INSTRON 3345 testing machine. Here the preparations included the setup of the testing machine and the drilling of a tiny hole through the ball's rubber wall. This was necessary to let the air leave from the ball during the compression, therefore we could distinguish the behavior

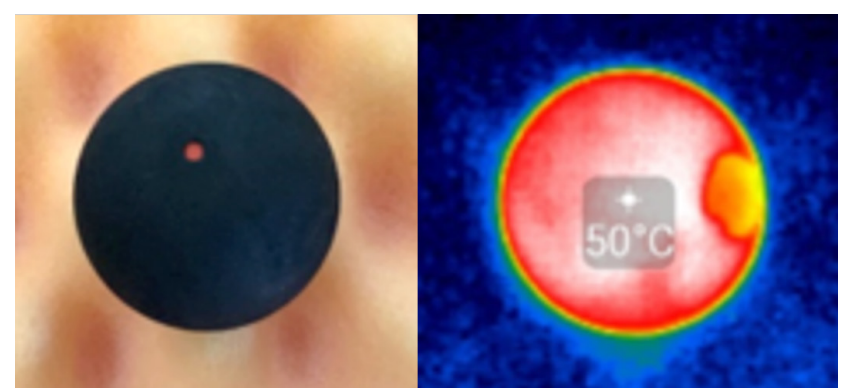

Fig. 2 Measuring the temperature of the squash ball's surface. The orange region on the right is a water droplet. 
of the balls' materials from the effect of the inner air. The entire loading cycle consisted three regimes:

1. compression with maximum displacement of $27.5 \mathrm{~mm}$

2. $10 \mathrm{~s}$ of holding

3. unloading.

The crosshead's velocity was $300 \mathrm{~mm} / \mathrm{min}$ in the loading and unloading phases. The relaxation part was applied to reveal the viscoelastic behavior of the balls in different conditions (unharmed, drilled).

\section{Results}

\subsection{Impact tests}

Fig. 3 visualizes a particular impact by its 8 frames recorded by the high-speed camera, where the upper ones from left to right belong to the ingoing ball and the lower ones represent the rebound phase from right to left according to the actual movement of the ball.

During the evaluation, we used only the horizontal component of the velocity while the change in the vertical component is negligible. The evaluated rebound velocities as a function of the initial velocity of the ball at three different temperature levels are shown in Fig. 4 in the case of the "fastest" ball (a) and "slowest" one (b).

The velocity difference between the balls is noticeable at the lower range but not too significant at higher velocities. On the other hand, the difference in the rebound velocity caused by the temperature change is remarkable. For example, in Fig. 4(b), the degree of the speed difference is around $5 \mathrm{~km} / \mathrm{h}$ per every $10^{\circ} \mathrm{C}$ in the range of $90-150 \mathrm{~km} / \mathrm{h}$. The reason for the velocity growth is the increased stiffness of the ball caused by the warm-up. An interesting fact that the rebound velocities belonging to the maximal initial values are lower than the ones belonging to the preceding initial speeds almost every time. From this observation, we can conclude that there is an optimal initial velocity distinct from the maximum one where the rebound velocity of the ball is higher than in all other cases.

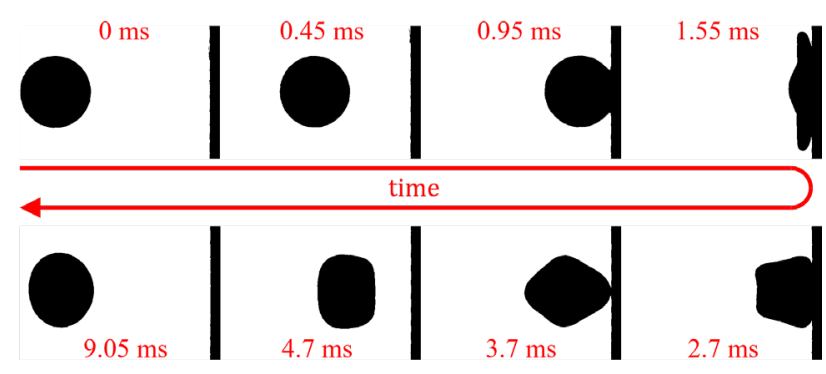

Fig. 3 Representation of an impact by its frames. The elapsed time is indicated.
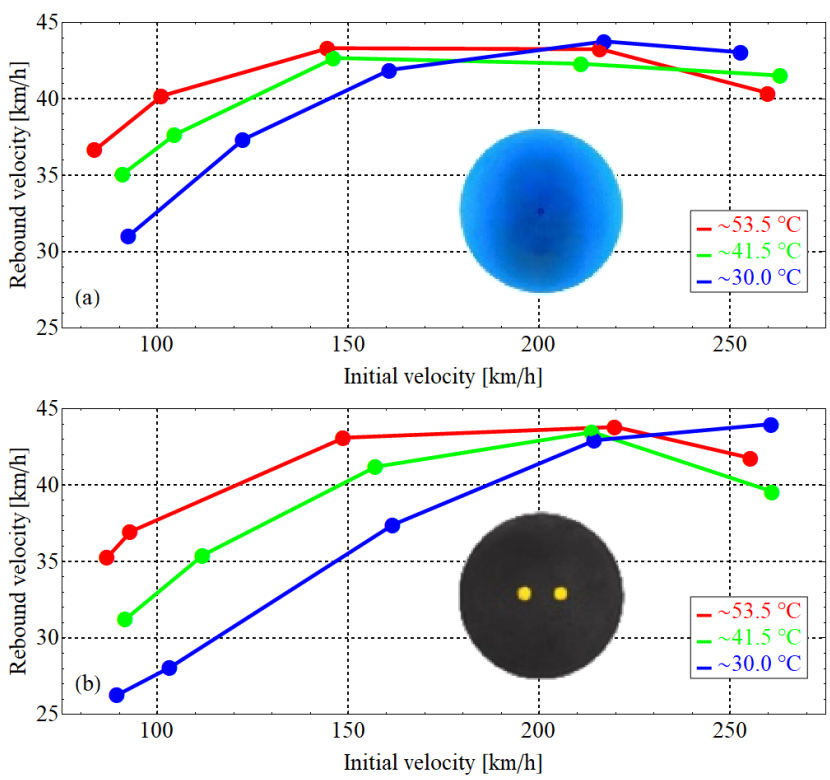

Fig. 4 Rebound velocity as a function of initial velocity; (a) "fastest" ball; (b) "slowest" ball

The impacts' two most important parameters are the coefficient of restitution (COR) and the contact time $\left(\mathrm{t}_{\mathrm{c}}\right)$. Let us investigate the COR at different ball types first, then the effect of the temperature change on this parameter. The definition of COR is the ratio of the rebounding and the ingoing velocities (see Eq. (1)).

The visual representation of the calculation is illustrated in Fig. 5 for room temperature.

As the initial speed grows, the COR will decrease - as we expected - because the higher velocity will result in larger deformations, which cause higher energy loss and lower rebounding velocity in proportion. The difference between the ball types is noticeable at the lower velocity range, unlikely to the higher range where it is barely visible. The "fastest" blue ball has the highest COR, as we thought from the investigation of the rebounding velocity.

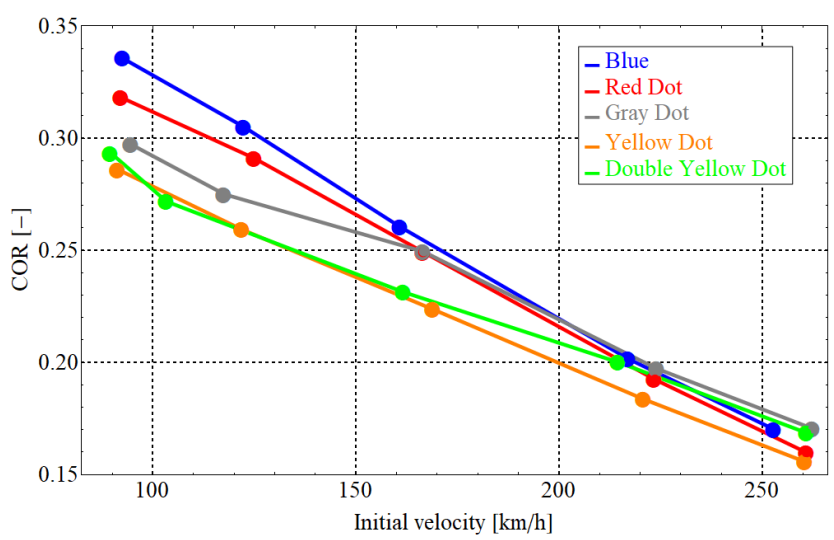

Fig. 5 Coefficient of restitution as a function of the initial velocity Comparing different ball types at room temperature 
$\mathrm{COR}=\frac{\mid \text { rebounding velocity } \mid}{\mid \text { ingoing velocity } \mid}$

The effect of the temperature on the COR is represented in Fig. 6 for the double yellow ball.

As we can see, the coefficient of restitution grows as the temperature increases, which means that the rebounding speed with the same ingoing velocity will be higher in the case of a heated ball than in the case of room-temperature. However, it should be noted this observation is not valid at very high velocities, where the ball's deformation is severe. On the other hand, the ball suffers greater deformations at higher temperature levels as Figs. 7 and 8 show. It causes this nontrivial phenomenon at the very high-velocity range ( above $220 \mathrm{~km} / \mathrm{h}$ ) where the ball at room temperature has higher rebound velocity than the same ball type with increased temperature (Fig. 4). This explains the fact why we experienced this contradiction in the impact behavior and higher COR value for the unheated ball after a certain velocity (Fig. 6).

Let us investigate the impacts' another important parameter which is the contact time. The contact period was that part of the video where contact was formed between the ball and the impact surface. The first frame is when

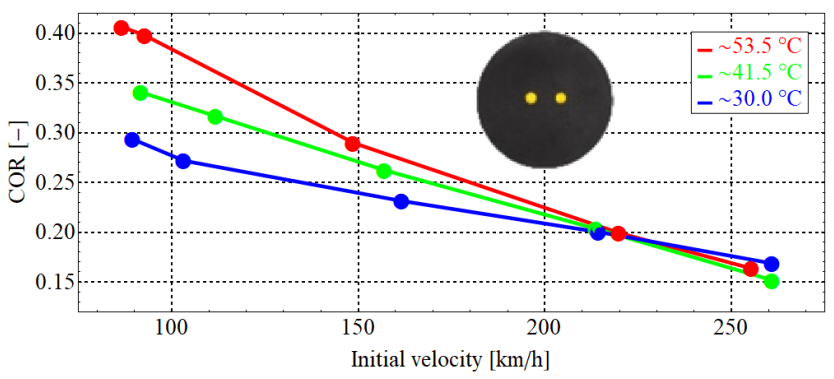

Fig. 6 Investigating the temperature's effect on the coefficient of restitution
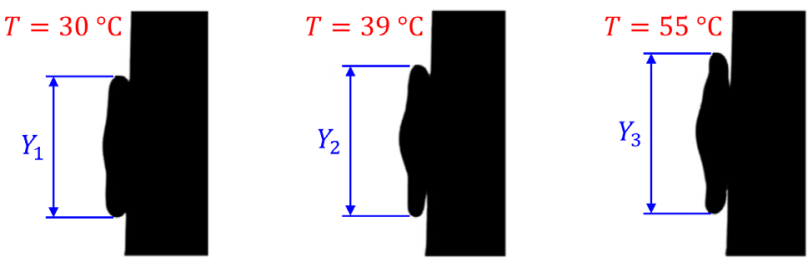

Fig. 7 Deformation of the blue ball horizontally to the wall at different temperatures
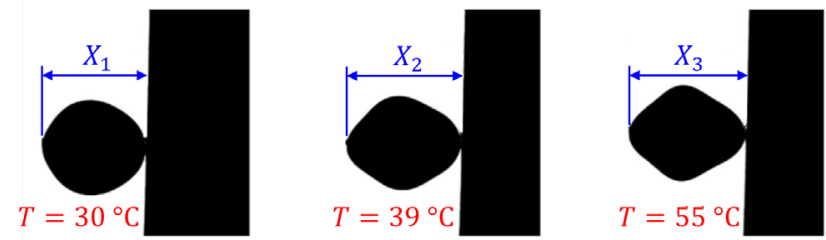

Fig. 8 Deformation of the blue ball perpendicularly to the wall at different temperatures the ball touched the wall, whereas the last frame is the particular one, where the ball lost the connection with the surface. Using the number of frames within these boundaries and the frame-rate, the contact time can be calculated. These periods of time as a function of the initial velocity are shown in Fig. 9 for every ball type at room-temperature.

As the ingoing speed grows, the contact time will be shorter, which met our expectations. The difference between the ball types becomes visible as the initial velocity increases in contrast to the COR where it was noticeable at the lower velocity range. The blue ball's contact time is the longest, while the double yellow dot ball has the shortest contact time period. It fulfills the expectations again as the blue ball belongs to the beginners and the double yellow dot to the professional players.

In Fig. 10, we can see that the heating of the ball results in a longer contact time. It can be explained by the fact that a heated ball during an impact suffers greater deformations than in the case of a ball at room temperature if we consider the same initial velocity.

To determine precisely these deformations, let us investigate the impacts with nearly the same initial speed but at different temperature levels. In Fig. 7, there are 3 frames from 3 different impacts with the blue ball where the horizontal deformation to the wall was maximal in all of the cases.

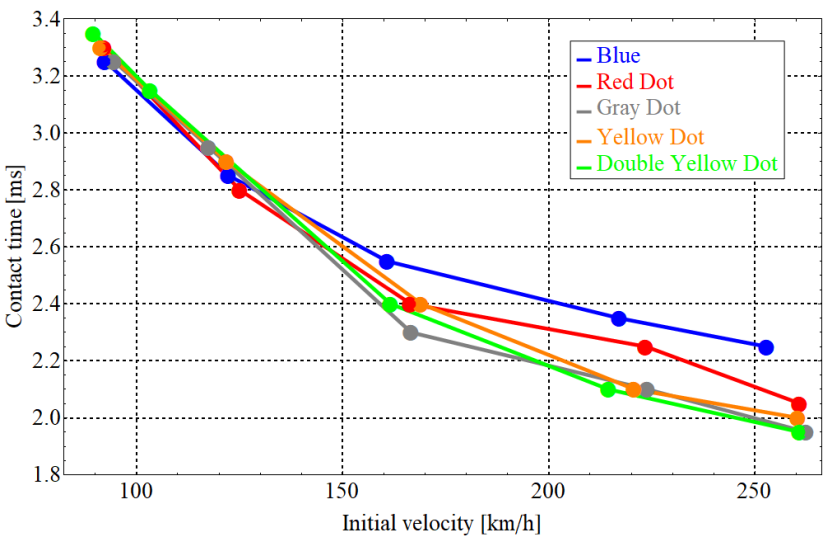

Fig. 9 Contact time as a function of the initial velocity - Comparing different ball types

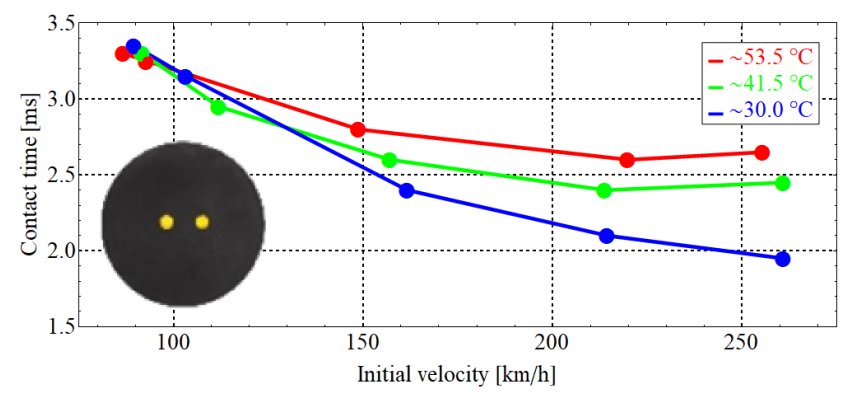

Fig. 10 Investigating the temperature's effect on the contact time 
The difference is clear, but it is more visible in the horizontal direction perpendicular to the wall directly at the moment before the contact was lost. These frames can be seen in Fig. 8.

The dimensions of the deformed shapes are summarized in Table 2 with the initial velocity and temperature values belonging to them.

The influence of the temperature change on the deformation is significant. Compared to the initial condition $\left(30^{\circ} \mathrm{C}\right)$ the $10^{\circ} \mathrm{C}$ temperature increase caused a $12 \%$ higher deformation while the $25{ }^{\circ} \mathrm{C}$ one caused $18 \%$ higher deformation in the vertical direction parallel to the wall in the case of the blue ball. To eliminate the effect of the slightly different initial speed values we show the same evaluation for the yellow dot ball in Table 3 .

In this case, we can see that the initial velocities are within a $1 \mathrm{~km} / \mathrm{h}$ range but the results are remarkable as well.

A reference by Cross [22] concluded that during an impact between a hollow ball and a flat surface, one could experience that the contact area which touches the wall first can buckle inwards. An example of this phenomenon can be seen in Fig. 11. However, if we would like to investigate this behavior, a camera setup perpendicularly to a plexi-glass apparatus would be a better solution to record the impact surface in detail.

After discussing the most important parameters, let us investigate the energy loss of the collision. One could read

Table 2 Blue ball's deformed dimensions at different temperatures

\begin{tabular}{lcccc}
\hline & $\begin{array}{c}\text { Initial velocity } \\
{[\mathrm{km} / \mathrm{h}]}\end{array}$ & $\begin{array}{c}\text { Temperature } \\
{\left[{ }^{\circ} \mathrm{C}\right]}\end{array}$ & \multicolumn{2}{c}{ Deformed dimensions } \\
\cline { 4 - 5 } & 252 & 30 & $\mathrm{Y}[\mathrm{mm}]$ & $\mathrm{X}[\mathrm{mm}]$ \\
\hline 1. & 263 & 39 & 60.9 & 43.2 \\
2. & 259 & 55 & 65.4 & 47.6 \\
3. & & & & 47.9 \\
\hline
\end{tabular}

Table 3 Yellow dot ball's deformed dimensions at different temperatures

\begin{tabular}{lcccc}
\hline & $\begin{array}{c}\text { Initial velocity } \\
{[\mathrm{km} / \mathrm{h}]}\end{array}$ & $\begin{array}{c}\text { Temperature } \\
{\left[{ }^{\circ} \mathrm{C}\right]}\end{array}$ & \multicolumn{2}{c}{ Deformed dimensions } \\
\cline { 4 - 5 } & 262 & 30 & $\mathrm{Y}[\mathrm{mm}]$ & $\mathrm{X}[\mathrm{mm}]$ \\
\hline 1. & 261 & 41 & 61.6 & 39 \\
2. & 261 & 53 & 66.6 & 46 \\
3. & & & & 47.3 \\
\hline
\end{tabular}
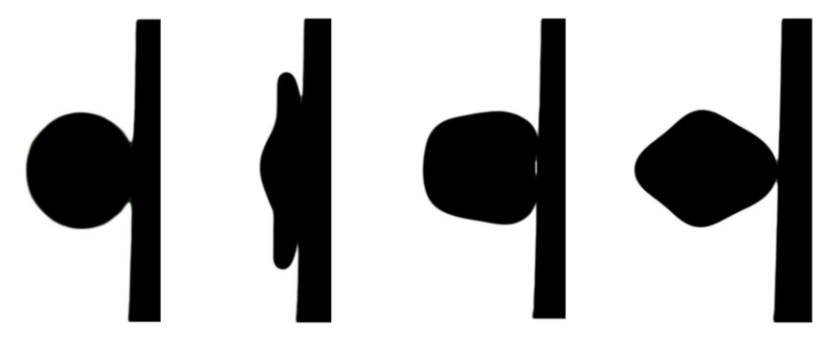

Fig. 11 Buckling phenomenon during impact a detailed article about this topic presented by Tadrist and Texier [24]. As a brief summary, we can state that most of the kinetic energy is dissipated due to the deformation of the ball during the impact. Some of the energy is stored in the ball as the increased internal air pressure and elastically in the rubber wall. The rest is lost as sound or as thermal energy in the impact surface, in the air or in the ball's wall. The reason for the heat generation is the friction between the impact surface and the ball and the internal friction of the rubber material itself. The increased temperature influences the mechanical properties of the ball's material as well as the internal pressure of the ball.

As we considered these impacts as planar motions, we utilized the simplified formula to calculate the kineticenergy. The kinetic energy of a rigid body is the sum of the translational part and the rotational part by definition as follows:

$E_{k}=\frac{1}{2} m v_{G}^{2}+\frac{1}{2} \theta_{G} \omega^{2}$,

where:

- $E_{k}$ : kinetic energy of the squash ball [ $\left.\mathrm{J}\right]$,

- $m$ : mass of the squash ball [kg],

- $v_{G}$ : velocity of the squash ball's center of gravity $[\mathrm{m} / \mathrm{s}]$,

- $\theta_{G}$ : squash ball's moment of inertia calculated for the center of gravity $\left[\mathrm{kgm}^{2}\right]$,

- $\omega$ : angular velocity of the squash ball $[\mathrm{rad} / \mathrm{s}]$.

The fact that the air-cannon should shoot the ball without remarkable angular momentum was one of the design requirements. As Fig. 12 shows, it satisfies this condition in a way that we can neglect the rotational part of the kinetic energy as the ball's angular velocity is nearly zero.

The more simplified term for the kinetic energy is the following:

$E_{k}=\frac{1}{2} m v_{G}^{2}$

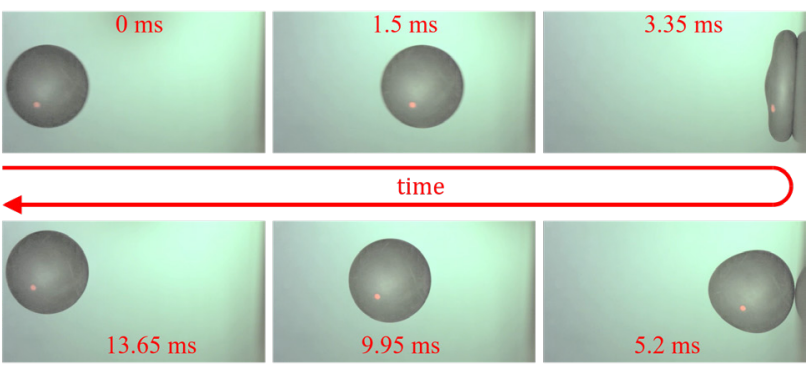

Fig. 12 Top row: impact phase from left to right; Bottom row: rebound phase from right to left according to the actual motion. The elapsed time is indicated. 
Using this formula, the kinetic energy after the impact as a function of the kinetic energy before the impact can be seen in Fig. 13 for all the ball types at room-temperature.

Our previous experience can be observed here as well. If the kinetic energy of the ball increases due to the initial velocity growth there will be bigger deformations which results in higher energy loss. The difference between the ball types are noticeable especially at the lower velocity range.

As we experienced it above, the temperature growth causes higher deformation and higher rebounding velocities in a dominant range of the investigated inbound velocities. However, the rebounding velocity can be smaller at higher inbound velocity at the same temperature value as it can be seen in in Fig. 4. This phenomenon can be also demonstrated by plotting the output kinetic energy versus the input kinetic energy (see Fig. 14)

The most important parameter which relates to the energy loss is the so-called rebound resilience which can be described as the material's ability to absorb energy when the object is loaded, then it releases this energy during the unloading phase [23]. This value can be calculated by the ratio of the kinetic energy after and before the impact, which is a really low value in terms of squash balls. The rebound resilience comparison of the ball types as a

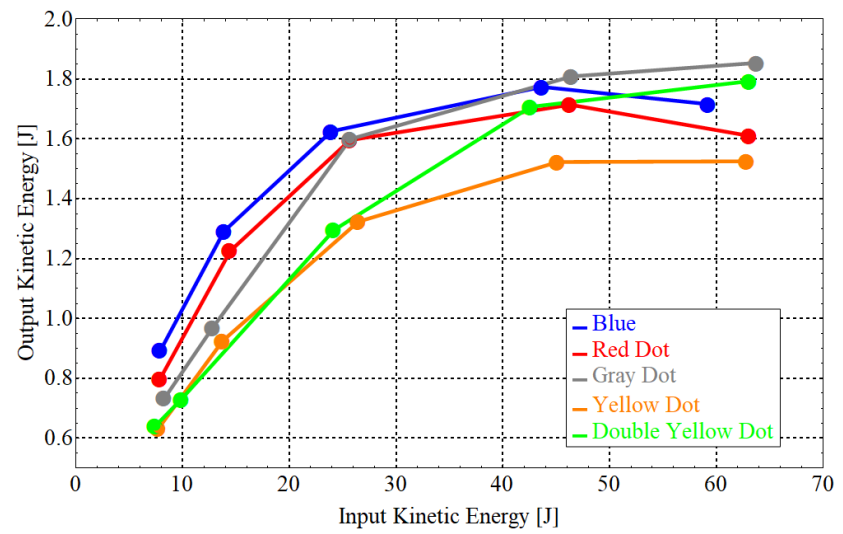

Fig. 13 Output kinetic energy as a function of the kinetic energy before the impact - Comparing different ball types

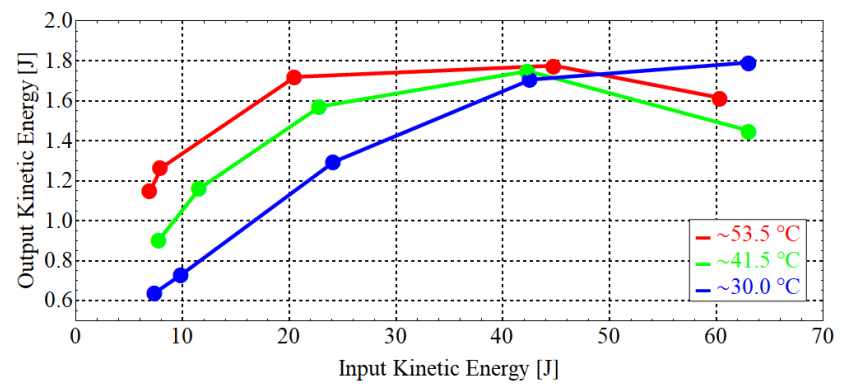

Fig. 14 Investigating the temperature's effect on the kinetic energy of the ball function of the initial velocity can be seen in Fig. 15 and the influence of the temperature on this parameter in the case of the double yellow dot ball is illustrated in Fig. 16.

In the results, we can see indeed that a huge amount of energy dissipated during the impact. The blue ball has the higher rebound resilience while the double yellow dot has the lower one. The growth of this parameter value caused by the temperature is also noticeable which met our expectations from the previous investigations.

\subsection{Compression tests}

The results of the compression test at room temperature can be seen below. Fig. 17 shows the compressive force results for the unharmed blue and double yellow dot balls. The force-displacement curves for the harmed balls (having tiny drilled holes) are presented in Fig. 18.

In these figures, we can distinguish the 3 phases of the load cycle. During the compression the acting force constantly increases by the displacement of the crosshead, then at the holding phase we can see the relaxation of the rubber. It can also be stated that during the compression the stiffer double yellow dotted ball needs higher acting force at the same displacement value compared to the blue ball. The relaxation behavior can be remarked mostly on

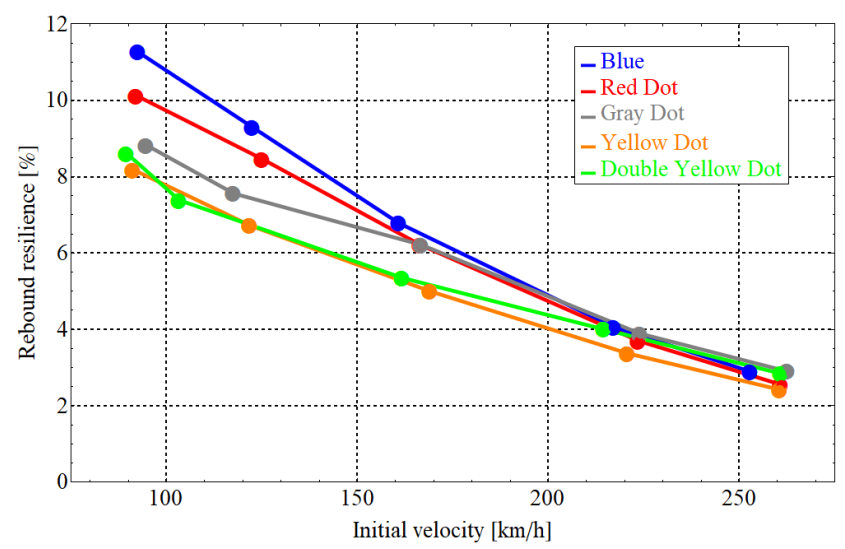

Fig. 15 Rebound resilience as a function of the initial velocity Comparing different ball types

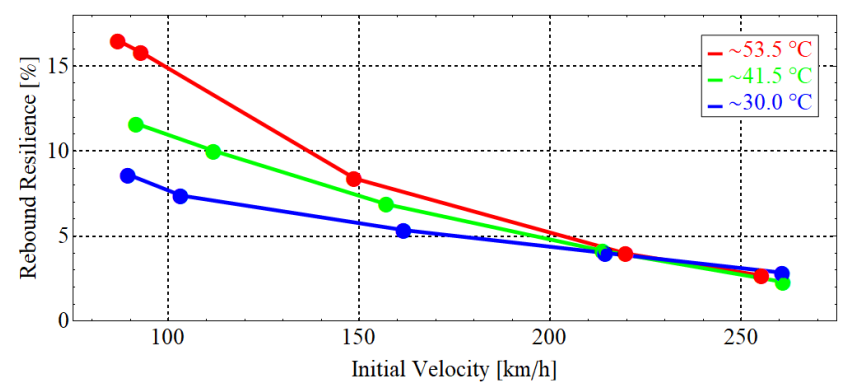

Fig. 16 Investigating the temperature's effect on the rebound resilience of the ball 


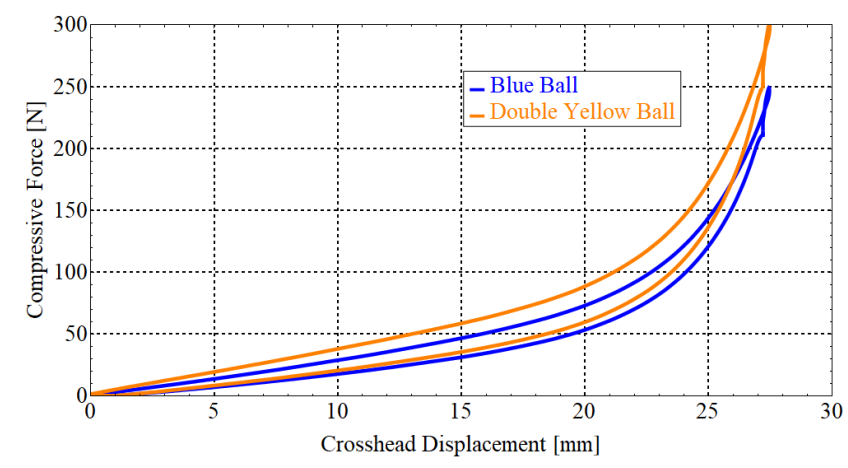

Fig. 17 Compression force versus displacement curves for the blue and the double yellow dot balls

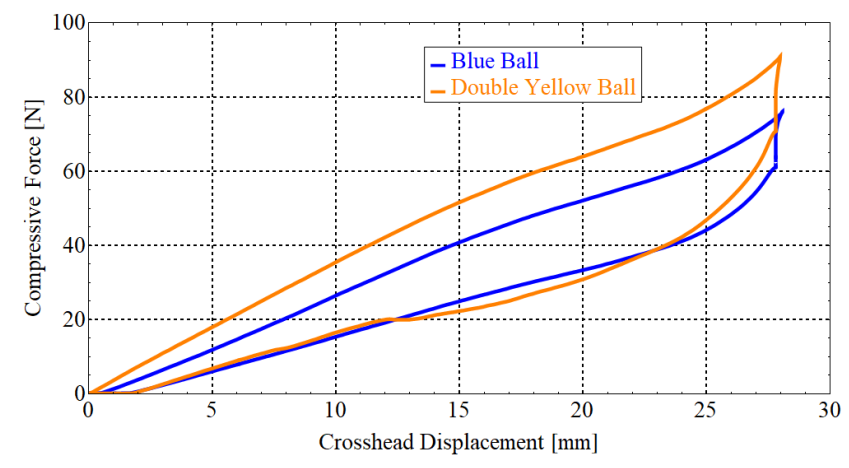

Fig. 18 Compression force versus displacement curves for the harmed blue and the double yellow dot balls

the drilled balls in the form of a hysteresis loop which has a larger area in the case of the double yellow dot ball. This is exactly the result what we expected based on the experiences from the impact tests, where this type had the smallest rebound resilience which meant the highest energy losses during the collisions. The difference between the stiffness of the unharmed and harmed blue balls is shown in Fig. 19.

In the beginning phase until around $10 \mathrm{~mm}$ crosshead displacement the two curves look almost identical which means that the inner air has no significant effect within this region. However after $15 \mathrm{~mm}$ the unharmed ball's curve has a rapid increase. It can be remarked that at the end of the process for the same amount of displacement

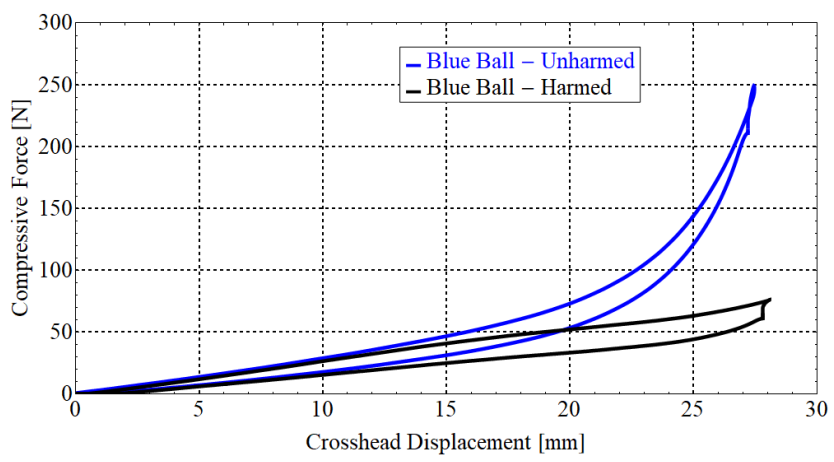

Fig. 19 Comparison of the loading curves of the unharmed and harmed ball we need about three times bigger compression force in the case of the unharmed ball than for the drilled one. In comparison to Lewis et al. [23], this result showed that the inner air could have a huge effect in the rebound behavior when we are considering large deformations. We can state that large deformation has already occurred above $90 \mathrm{~km} / \mathrm{h}$ which is a usual ball speed during a real squash match. An example for the blue ball at room temperature can be seen in Fig. 20, with an initial speed of $92 \mathrm{~km} / \mathrm{h}$. An image of the compression test at the lowest position is also illustrated to make the deformations comparable.

Finally, in Fig. 21, we can see the effect of the warming up process on the characteristics of the blue ball.

In the diagram we can see 3 cases, namely the specimen in room temperature, one heated up to $50{ }^{\circ} \mathrm{C}$ which represents a realistic case in terms of a squash game, then the last one which was warmed up to $90{ }^{\circ} \mathrm{C}$ to make the difference noticeable. As we can see, for the same amount of compression we need a higher compression force in the heated cases. This means that the balls harden, their overall stiffness becomes greater. This result is consistent with our observations in the impact tests where we experienced higher impact velocities as the balls were warmed up.

\section{Conclusions}

From the measurements and the evaluation process, we can conclude that the observed parameters mainly changed in

\section{Compression test}

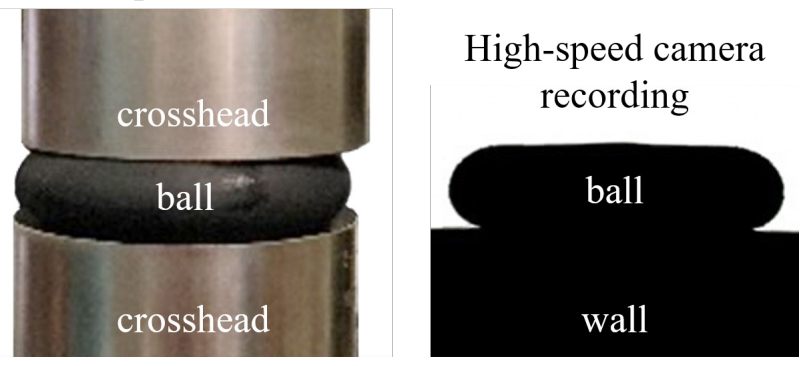

Fig. 20 Comparison of the deformed ball in compression test (left) and in the impact test (right)

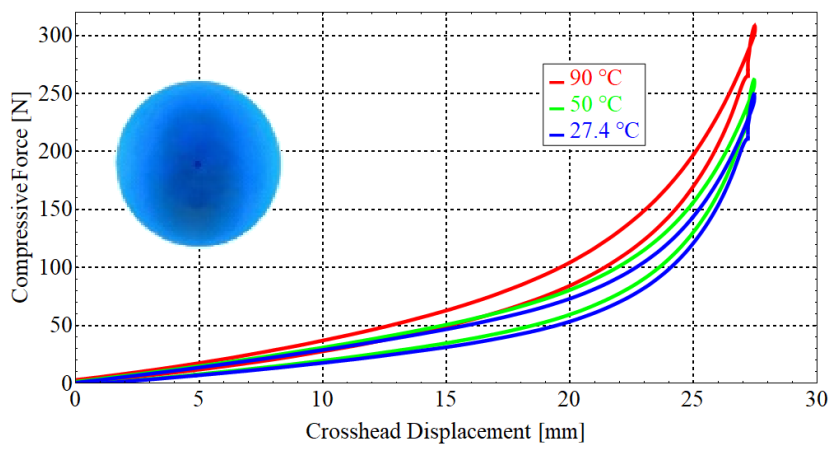

Fig. 21 Effect of the temperature on the ball's compression characteristics 
response to the temperature change in different ball types as we expected from the articles which dealt with this topic previously. We found evidences for some interesting phenomena presented before while we experienced some new ones which raised more questions.

In general, we can say that the COR, the contact time and the rebound resilience will decrease with the increase of the initial velocity, while these parameters will grow by the increase of the temperature of the ball. What is really important, that in our case these statements are not valid for the very high velocity range. An unexpected event was, that using this given product, there is an optimal initial velocity distinct from the maximum one where the rebound velocity of the ball is higher than in all other cases. Considering that the velocity range used during the measurements met the velocity range occurring at a real squash game, we can say this is a valuable experience.

From the results of the impact test we also found an evidence for an interesting buckling phenomenon of the ball which was mentioned by Cross [22] before.

We can conclude that within the low velocity range when the ball suffers smaller deformations the ball's material dominates and almost all the compression force arises from the deformation of the rubber. However, in contrast of Lewis et al. [23], we state that the internal air pressure has a huge effect on the rebound behavior when we are considering large deformations, which is usual during a squash ball game where the ball's velocity is mainly above $100 \mathrm{~km} / \mathrm{h}$.

The compression test results were consistent with our experiences came from the impact test e.g. in case of the double yellow dotted ball, the area of the hysteresis loop was bigger compared to the blue ball which explains the lower rebound resilience value as well as the lower impact velocity.

\section{References}

[1] Arakawa, K., Mada, T., Komatsu, H., Shimizu, T., Satou, M., Takehara, K., Etoh, G. "Dynamic Deformation Behavior of a Golf Ball during Normal Impact", Experimental Mechanics, 49(4), pp. 471-477, 2009

https://doi.org/10.1007/s11340-008-9156-y

[2] Arakawa, K., Mada, T., Komatsu, H., Shimizu, T., Satou, M., Takehara, K., Etoh, G. "Dynamic Contact Behavior of a Golf Ball during Oblique Impact: Effect of Friction between the Ball and Target", Experimental Mechanics, 47(2), pp. 277-282, 2007. https://doi.org/10.1007/s11340-006-9018-4

[3] Arakawa, K., Mada, T., Komatsu, H., Shimizu, T., Satou, M., Takehara, K., Etoh, G. "Dynamic Contact Behavior of a Golf Ball during an Oblique Impact”, Experimental Mechanics, 46(6), pp. 691-697, 2006. https://doi.org/10.1007/s11340-006-9257-4
It is really hard to describe such a complex dynamical behavior as the impact of a squash ball. To summarize the experiences we can state from both the impact and compression tests that the ball's overall stiffness grows as the temperature increases. On the other hand, the ball suffers greater deformations at higher temperature levels as Figs. 7 and 8 showed earlier, which caused some nontrivial phenomena at the very high-velocity range. To investigate more thoroughly the effects of the viscoelastic material behavior and the internal air pressure, one can present an analysis with the usage of an accurate material model and finite element method. That may explain the contradiction in the impact behavior in detail.

Overall, we think that such a comprehensive summary of squash ball impacts has not been published in the literature yet. The results presented in this paper give a larger insight into the dynamics of squash balls and they could be useful at the investigation of other similar phenomena as well.

\section{Acknowledgment}

This research was supported by the János Bolyai Research Scholarship of the Hungarian Academy of Sciences; Hungarian National Research, Development and Innovation Office (NKFI FK 128662); the ÚNKP20-5 New National Excellence Program of the Ministry for Innovation and Technology from the source of the National Research, Development and Innovation Fund. The research reported in this paper and carried out at BME has been supported by the NRDI Fund (TKP2020 IES, Grant No. BME-IE-NAT and TKP2020 NC, Grant No. BME-NC) based on the charter of bolster issued by the NRDI Office under the auspices of the Ministry for Innovation and Technology.

[4] Ball, K., Hrysomallis, C. "Synthetic grass cricket pitches and ball bounce characteristics", Journal of Science and Medicine in Sport, 15(3), pp. 272-276, 2012.

https://doi.org/10.1016/j.jsams.2011.10.010

[5] Peploe, C., McErlain-Naylor, S. A., Harland, A. R., King, M.A. "The relationships between impact location and post-impact ball speed, bat torsion, and ball direction in cricket batting", Journal of Sports Sciences, 36(12), pp. 1407-1414, 2018. https://doi.org/10.1080/02640414.2017.1389484

[6] Axe, J. D., Brown, K., Shannon, K. "The vibrational mode structure of a golf ball", Journal of Sports Sciences, 20(8), pp. 623-627, 2002. https://doi.org/10.1080/026404102320183185

[7] Cross, R. "Impact behavior of a superball", American Journal of Physics, 83(3), pp. 238-248, 2015. https://doi.org/10.1119/1.4898312 
[8] Cross, R. "Behaviour of a bouncing ball", Physics Education, 50(3), pp. 335-341, 2015.

https://doi.org/10.1088/0031-9120/50/3/335

[9] Laird II, G. "An investigation of ball-on-ball impact", Experimental Mechanics, 29(3), pp. 300-306, 1989. https://doi.org/10.1007/BF02321412

[10] Cross, R. "Oblique Bounce of a Rubber Ball", Experimental Mechanics, 54(9), pp. 1523-1536, 2014. https://doi.org/10.1007/s11340-014-9938-3

[11] Cross, R. "Enhancing the Bounce of a Ball", The Physics of Teacher, 48(7), pp. 450-452, 2010. https://doi.org/10.1119/1.3488187

[12] Rezaei, A., Verhelst, R., Van Paepegem, W., Degrieck, J. "Finite element modelling and experimental study of oblique soccer ball bounce", Journal of Sports Sciences, 29(11), pp. 1201-1213, 2011. https://doi.org/10.1080/02640414.2011.587443

[13] Bao, R. H., Yu, T. X. "Collision and rebound of ping pong balls on a rigid target", Materials \& Design, 87, pp. 278-286, 2015. https://doi.org/10.1016/j.matdes.2015.08.019

[14] Cross, R. "Impact of a ping-pong ball", Physics Education, 52(3), Article number: 033002, 2017. https://doi.org/10.1088/1361-6552/aa5f84

[15] Zhang, X. W., Tao, Z., Zhang, Q. M. "Dynamic behaviors of visco-elastic thin-walled spherical shells impact onto a rigid plate", Latin American Journal of Solids and Structures, 11(14), pp. 2607-2623, 2014. https://doi.org/10.1590/S1679-78252014001400009

[16] Brody, H. "Bounce of a tennis ball", Journal of Science and Medicine in Sport, 6(1), pp. 113-119, 2003. https://doi.org/10.1016/S1440-2440(03)80014-2

[17] Cant, O., Kovalchik, S., Cross, R., Reid, M. "Validation of ball spin estimates in tennis from multi-camera tracking data", Journal of Sports Sciences, 38(3), pp. 296-303, 2020. https://doi.org/10.1080/02640414.2019.1697189

[18] Goodwill, S. R., Haake, S. J. "Ball spin generation for oblique impacts with a tennis racket", Experimental Mechanics, 44(2), pp. 195-206, 2004.

https://doi.org/10.1177/0014485104039763
[19] Goodwill, S. R., Kirk, R., Haake. S. J. "Experimental and finite element analysis of a tennis ball impact on a rigid surface", Sports Engineering, 8, pp. 145-158, 2005.

https://doi.org/10.1007/BF02844015

[20] Haake, S. J., Carré, M. J., Goodwill, S. R. "The dynamic impact characteristics of tennis balls with tennis rackets", Journal of Sports Sciences, 21(10), pp. 839-850, 2003. https://doi.org/10.1080/0264041031000140329

[21] Liu, Y. K. "Mechanical analysis of racket and ball during impact", Medicine \& Science in Sports \& Exercise, 15(5), pp. 388-392, 1983. https://doi.org/10.1249/00005768-198315050-00008

[22] Cross, R. "Impact behavior of hollow balls", American Journal of Physics, 82(3), pp. 189-195, 2014. https://doi.org/10.1119/1.4839055

[23] Lewis, G. J., Arnold, J. C., Griffiths, I. W. "The dynamic behavior of squash balls", American Journal of Physics, 79(3), pp. 291-296, 2011. https://doi.org/10.1119/1.3531971

[24] Tadrist, L., Texier, B. D. "Open questions on the impact of an inflated ball", [cond-mat.soft], arXiv:1708.01282, Cornell University, Ithaca, NY, USA. [online] Available at: https://arxiv. org/abs/1708.01282 [Accessed: 06 March 2019]

[25] Cunnington, J. "Determining the mathematical relationship between the thermal energy applied to a squash ball and the consequential gain in hang time of the ball", Science One Research Projects, University of British Columbia, Vancouver, Canada, 2009. https://doi.org/10.14288/1.0107207

[26] World Squash Federation "World Squash, Singles Rules 2020", [pdf] World Squash Federation Ltd, Hastings, East Sussex, UK, Available at: https://www.worldsquash.org/rules-of-squash-2/ [Accessed: 17 April 2021] 